\title{
A young soccer player with sudden pain after kicking the ball
}

\author{
Sara Lega trainee paediatrician, Ingrid Rabach medical doctor, Egidio Barbi medical doctor, \\ Alessandro Ventura professor of paediatrics
}

Institute for Maternal and Child Health, Burlo Garofolo, Trieste, 34137, Italy

A 14 year old boy presented to the emergency department with a 10 day history of pain in his left hip. He reported that the pain had started after he kicked the ball during a soccer game. The pain was not particularly intense-he played until the end of the match—but it had persisted since then, tended to recede at rest, and reappeared during soccer training.

No swelling or bruising was seen on inspection of the inguinal area. He could walk and bear weight on his left leg without pain. Moderate tenderness was referred to the inguinal region, slightly below the inguinal ligament. Movements of flexion and lateral rotation of the hip were limited because of pain. A diagnosis of pelvic muscular strain was made and he was discharged with anti-inflammatory drugs and rest for one week. He was initially well but the pain recurred when he began to play soccer one week later. Plain radiography was then performed (fig 1).

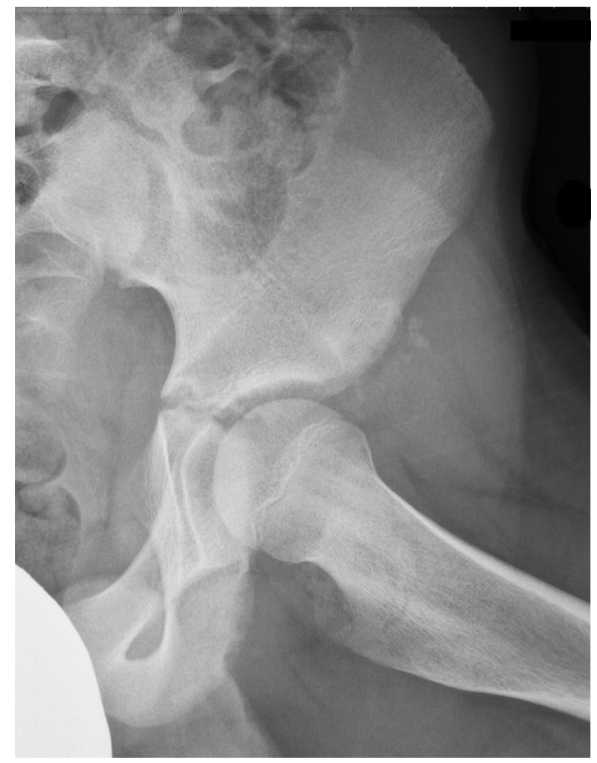

\section{Questions}

1. What does the radiograph show?

2. What is the diagnosis?

3. How does this condition usually present?

4. What are the treatment options?

5. What is the prognosis?

\section{Answers}

\section{What does the radiograph show? Answer}

A bone fragment displaced from the pelvis.

\section{Discussion}

Plain radiography of the pelvis shows a $1 \mathrm{~cm}$ bony fragment displaced laterally to the pelvic bone (fig 2). A subtle cortical irregularity of the pelvis is also visible, which suggests that the fragment originated from the anterior inferior iliac tuberosity. 


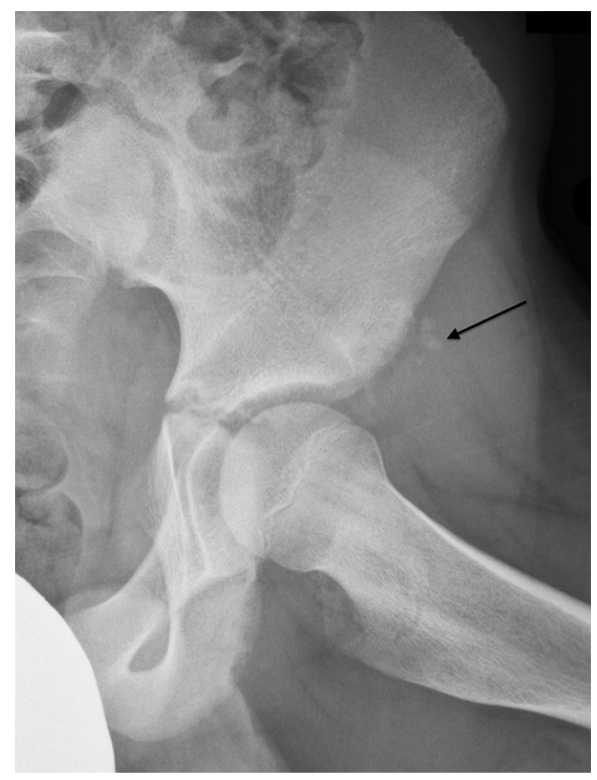

Fig 2 Pelvic radiograph showing a bone fragment (about $1 \mathrm{~cm}$ ) displaced laterally to the pelvic bone (arrow)

A conventional anteroposterior radiograph is the imaging modality of choice when a pelvic fracture is suspected: displaced bone fragments are usually seen as radiopaque particles lying immediately adjacent to the parent bone. However, when the fragment is only minimally displaced it may be obscured by the underlying bony pelvis. For this reason, when there is clinical suspicion of bone detachment, oblique or axial projections should also be performed. ${ }^{1}$ Furthermore, when interpreting pelvic radiographs in children and adolescents, the presence of areas of incomplete ossification should be taken into account. The avulsed fragment may be a part of the pelvis that is not yet ossified and not visible radiographically.

In the hands of a skilled operator, sonography is an accurate and cost effective option when conventional radiography fails to show a clinically suspected avulsion. Four ultrasonographic criteria have been proposed:

- A hypoechoic zone in the region of the apophysis that extends to the surrounding soft tissue, which represents oedema or haemorrhage

- Widening of the normally hypoechoic physis between the apophysis and the pelvis, which represents lysis

- Dislocation of the apophysis, which represents avulsion

- Mobility of the apophysis on dynamic examination. ${ }^{23}$

Magnetic resonance imaging (MRI) can demonstrate the avulsed fragment and provide anatomical details of associated musculotendinous injuries without exposing the patient to ionising radiation. ${ }^{4}$

\section{What is the diagnosis?}

\section{Answer}

An apophyseal avulsion fracture of the left anterior inferior iliac spine.

\section{Discussion}

The bony fragment seen in the radiograph represents an avulsed ossification centre, known as an apophysis. Apophyses are secondary ossification centres found throughout the growing skeleton that contribute to the circumferential growth of the bone and serve as attachment sites for muscles and tendons.
Apophyses arise as separate ossification centres, being initially cartilaginous, and progressively ossify and fuse to the parent bone later in development. Before fusion, the apophysis is a weak point in the skeletal system, and any acute or repetitive forceful contraction of the muscle can pull it away from its growth plate. The most important predisposing factor is sports activity, particularly sports that involve sprinting, rapid changes in movement, or jumping, such as soccer, gymnastics, or tennis. Avulsion fractures can occur anywhere in the immature skeleton, but most often in the pelvis. ${ }^{5}$ Incidence is highest between puberty, when ossification of the pelvic apophyses begins, and 25 years-the age at which the apophyses fuse to the corresponding tuberosities. $^{67}$

The most common site of pelvic avulsion (table $\Downarrow$ ) is the ischial tuberosity, followed by the anterior inferior iliac spine, and then the anterior superior iliac spine. The table and fig 3 show the other possible avulsion sites on the pelvis, along with their muscle attachments and time of physis appearance and fusion. Avulsion at the anterior inferior iliac spine and anterior superior iliac spine occurs mostly in soccer players and sprinters because kicking and sprinting both require maximal exertion of the rectus femoris and sartorius muscles. Injuries to the ischial tuberosity are more common in gymnasts and are generally related to excessive lengthening of the hamstring muscles. Avulsion fractures were rarely reported in the past, but with the increasing participation of adolescents in competitive sports and improvements in imaging techniques they are increasingly being recognised. Pelvic injuries account for $10-24 \%$ of acute injuries in children and adolescents who take part in sports, ${ }^{8}$ and pelvic avulsions are the most common type of pelvic fracture in this age group. ${ }^{9}$

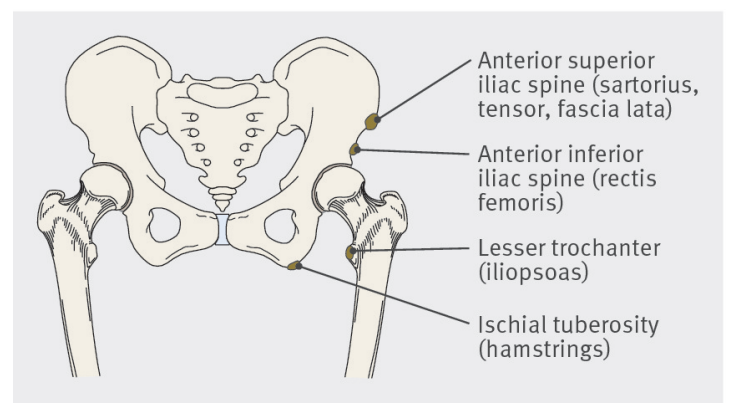

Fig 3 Possible sites of pelvic avulsion

\section{How does this condition usually present? Answer}

Presenting symptoms are acute onset of pain and restricted range of motion.

\section{Discussion}

History and physical examination are the key to diagnosis, with pain being the most common presenting symptom. Patients can usually recall the exact moment when the pain began, typically while playing sports. The pain may be localised to the involved tuberosity and usually results in restricted active motion,

weakness, limping, and local tenderness at the site of the injured apophysis.

Less painful clinical presentations are possible, particularly when the anterior inferior iliac spine is affected. ${ }^{4}$ Because the anterior inferior iliac spine is located deep in the pelvis, the pain may be modest, as in our case, and the area of tenderness may be difficult to pinpoint. Moreover, symptoms may appear only with contraction of the muscle attached to the injured apophysis 
(Duncan-Ely test: passive flexion of the knee while the patient lies prone).

Avulsion fractures may arise from a pre-existing inflammatory process, known as apophysitis, which can occur in competitive athletes during intensive training. These patients may experience pain of several weeks duration and may continue to train despite the pain. In this setting, avulsion fractures can be misdiagnosed as insertional tendinitis or musculotendinous tear and may be missed on initial presentation. ${ }^{10}$ When diagnosis is delayed, a mass of new bone may develop in the groin or buttock area due to heterotopic ossification. In such cases, a bone tumour would be included in the differential diagnosis. ${ }^{11}$ A history of trauma, together with physical examination and magnetic resonance imaging findings, should help to rule out the presence of a tumour.

\section{What are the treatment options?} Answer

A conservative approach that consists of rest and analgesics is most appropriate —only rare cases require surgical reattachment.

\section{Discussion}

Radiological examination facilitates evaluation of the size of the avulsed fragment and the degree of bony displacement. In most cases, a conservative approach-consisting of rest, application of ice, and analgesics followed by protected weight bearing with crutches until symptoms resolve (usually within three weeks) - is preferred. Isometric and progressive resistance exercise together with stretching can then be started. Patients can return to sports activities once they regain full strength of the affected muscles and full range of motion. However, to prevent re-injury, patients should not return to sports earlier than two months after the initial injury. ${ }^{12}$ Surgical intervention is rarely needed and is considered only when the avulsed fragment is in one piece. Indications for surgery are persistence of symptoms despite conservative treatment or large displacements $(>2 \mathrm{~cm})$, which are at risk of non-union and permanent loss of muscle strength. ${ }^{14}$

\section{What is the prognosis? \\ Answer}

Prognosis is excellent in most cases.

\section{Discussion}

When managed appropriately, most apophyseal avulsions heal with excellent results. Complications are rare, with the most common being painful non-union and heterotopic ossification. The risk of developing complications increases if activity continues after injury, diagnosis is delayed, or the advice to rest is ignored. Prognosis also depends on the site of avulsion. Non-union at the anterior inferior iliac spine and anterior superior iliac spine does not seem to affect muscle functioning. Most cases of persistent disability, with limitation to participation in sports and persistence of symptoms, have been reported after fractures of the ischium. Both neurological symptoms as a result of sciatic nerve impingement and bone and muscle atrophy of the posterior thigh due to overgrowth of the injured tuberosity have been seen after ischial avulsions. Painful non-unions and bone overgrowth can be managed with surgical treatment - both reattachment and excision of the fragment are commonly undertaken. ${ }^{12}$

\section{Patient outcome}

The boy was managed conservatively and returned to training after three months with no residual symptoms.

Competing interests: We have read and understood BMJ policy on declaration of interests and declare that we have none.

Provenance and peer review: Not commissioned; externally peer reviewed.

Parental consent obtained.

1 McKinney BI, Nelson C, Carrion W. Apophyseal avulsion fractures of the hip and pelvis. Orthopedics 2009;32:42.

2 Lazović D1, Wegner U, Peters G, et al. Ultrasound for diagnosis of apophyseal injuries. Knee Surg Sports Traumatol Arthrosc 1996;3:234-7.

3 Pisacano RM, Miller TT. Comparing sonography with MR imaging of apophyseal injuries of the pelvis in four boys. AJR Am J Roentgenol 2003;181:223-30.

4 Kjellin I, Stadnick ME, Awh MH. Orthopaedic magnetic resonance imaging challenge: apophyseal avulsions at the pelvis. Sports Health 2010;2:247-51.

5 Rossi F, Dragoni S. Acute avulsion fractures of the pelvis in adolescent competitive athletes: prevalence, location and sports distribution of 203 cases collected. Skeletal Radiol 2001;30:127-31.

6 Bylak J, Hutchinson MR. Common sports injuries in young tennis players. Sports Med 1998;26:119-32.

7 El-Khoury GY, Brandser EA, Kathol MH, et al. Imaging of muscle injuries. Skeletal Radiol 1996;25:3-11.

8 Boyd KT, Peirce NS, Batt ME. Common hip injuries in sports. Sports Med 1997;24:273-88

9 Ortega HW1, Reid S, Velden HV, et al. Patterns of injury and management of children with pelvic fractures at a non-trauma center. J Emerg Med 2014;47:140-6.

10 Pointinger H1, Munk P, Poeschl GP. Avulsion fracture of the anterior superior iliac spine following apophysitis. Br J Sports Med 2003;37:361-2.

11 Dhinsa BS1, Jalgaonkar A, Mann B, et al. Avulsion fracture of the anterior superior iliac spine: misdiagnosis of a bone tumour. J Orthop Traumatol 2011;12:173-6.

12 Naudé M, Lindeque BGJ, Rensburg DCJ. Avulsion fractures of the pelvis. South Afr J Sports Med 2003;15:18-24. 


\section{Table}

\section{Table 1| Pelvic avulsion: most common sites of avulsion and characteristics}

\begin{tabular}{|c|c|c|c|}
\hline ASIS & $13-15 \quad 21-25$ & Sartorius, tensor fascia lata & Soccer, athletics, gymnastics \\
\hline Alls & $13-15 \quad 16-18$ & Rectus femoris & Soccer, athletics, tennis \\
\hline Ischial tuberosity & $13-15 \quad 20-25$ & Hamstring & Gymnastics, soccer, tennis, running \\
\hline Lesser trochanter of the femur & $11-12 \quad 16-17$ & Iliopsoas & Athletics \\
\hline
\end{tabular}

AIIS=anterior inferior iliac spine; ASIS=anterior superior iliac spine. 